\title{
Machine Vision Based Plant Disease Classification Through Leaf Imagining
}

\author{
Hamza Ur Rehman*, Shahzad Anwar, Muhammad Tufail \\ Department of Mechatronics Engineering, University of Engineering and Technology, Peshawar 25000, Pakistan
}

Corresponding Author Email: 13pwmct0328@uetpeshawar.edu.pk

\section{https://doi.org/10.18280/isi.250405}

Received: 7 June 2020

Accepted: 2 August 2020

\section{Keywords:}

machine learning, multi-class SVM, machine vision

\begin{abstract}
Plants disease identification plays a major role in agriculture yield prevention. Traditionally, manual plant surface examination is conducted which is time-consuming and relatively less efficient. Therefore, this study incorporates machine vision-based techniques, for plant disease identification (i.e. healthy leaf, Alternaria Alternate, and bacterial blight). The developed method employs a dataset comprised of more than 10,000 data points. Initially, Image processing is performed followed by image pre-processing techniques for noise removal. Subsequently, image segmentation is performed for the region of interest (ROI). A multiclass classifier is introduced for plant disease analysis, which results in a percentage of disease spreading in healthy leaf. The results were compared with other methods and it is evident that the developed method has shown 95\% accuracy in plant disease identification.
\end{abstract}

\section{INTRODUCTION}

Modern technology has assisted farmers in meeting society's global food demands. Food security and crop health monitoring remain threatened by many factors, such as, climate change [1], pollinators reduction [2], and crop/plant diseases. A common medium of plant disease is microorganisms and insects. These micro-organisms leave visual clues on plant surfaces which could assist in plant disease detection. It is to note that studying diseases affecting plants caused due to bacterial or viral substances, lead to classify two subbranches of diseases such as (i) abiotic and (ii) biotic.

Abiotic in contrast, are caused by non-living ecological conditions and circumstances, such as chemicals, hail, weather condition etc. These diseases are non-infectious and nontransmissible and cause less harm compared to biotic plant diseases. On the other hand, Biotic plant diseases are caused by living organisms, such as viruses, fungi, and bacteria. Thus, disease plant prevention, yield loss, and plant health monitoring have shown great scope for researchers and is currently an expanding area of research. Previously, a common practice was to observe the leaf of a plant via the naked eye. This practice was mostly carried out by botonny experts, in international or national agriculture institutions and /or in agricultural extension organizations. This practice often requires expertise in botany, having the knowledge and experience to determine the state of the plant or a team of professional/experienced experts for continuous examination of the field. This practice is expensive as well as timeconsuming depending on the size of the field/farm. Thus, various efforts were made to develop a cost and time effective method to counter this problem [3].

With the development in the computational system over the past decade, especially, in embedded processors, Artificial Intelligence (AI) and machine learning have shown to be growing exponentially. Manual inspection is a timeconsuming and expensive task to oversee various things, such as plant health monitoring and disease. A dataset needs to be established to employ machine learning principals for plant disease detection. The dataset is required to be large and contains a variety of data points, having both diseased and healthy samples. The dataset is then provided to a supervised or unsupervised machine learning classifier that trains in terms of negative ( 0 ) and positive (1) data points provided in the database. The classifier finds abnormities on the surface of the leaf such as, brown spots as shown in Figure 1.

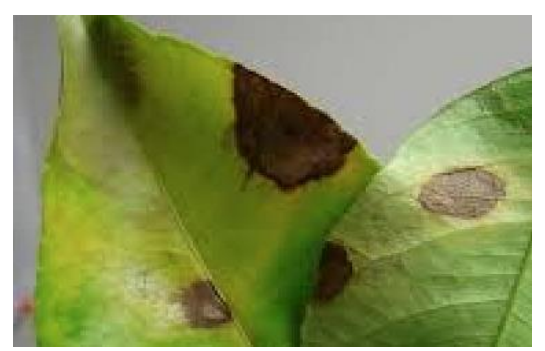

Figure 1. Alternaria alternate

Some common biotic disease is shown in Figure 2 which represent bacterial, viruses, and fungal diseases.

This table represents the most common symptoms of bacterial, fungal, and viral diseases that could be observed visually, however, to determine if it is a bacterial fungal or viral disease require tests.

While studying disease leaf, it was found the disease slowly covers the leaf by eliminating healthy cells in the leaf and slowly spreading over the leaf and ultimately the plant. However, a dying or dead leaf has part of it dying but has an overall effect. The leaves would turn colours, from red to yellow to purple, and then to brown. The skin and stem of the leaf become brittle and the leaf would then fall from the tree. The main visual clue for disease and dead leaf is the way it covers the leaf. The cellular examination, whether disease or dead cells, could be discovered after running tests. 


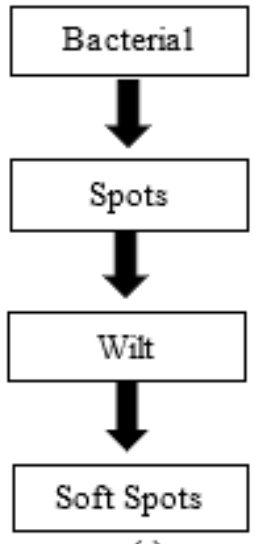

(a)

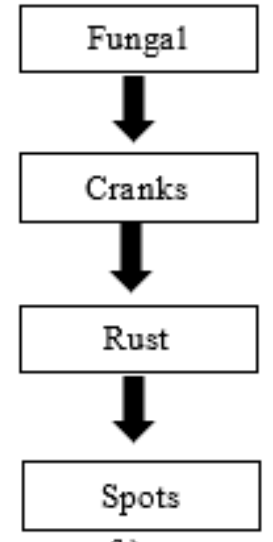

(b)
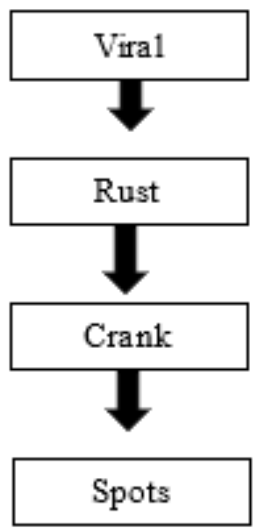

(c)
Figure 2. (a) Bacterial Disease, (b) Viral Disease, (c) Fungal Disease

According to a study [4], billions of dollars have been lost due to plant diseases and have caused heavy crop losses in Ireland. Approximately 1.5 million people deceased due to starvation leaving 1.5 million to relocate to other parts of the region. Renato et al. [5] in a study discussed that about more than 2 million plants were abolished from the lands of nurseries and farms due to various plant diseases. This resulted in an estimate of USD $\$ 116$ million spent for abolishing those diseases, infected trees, and plants in the past ten years still leaving its presence in the US. These unpleasant incidents have made plant disease detection and management a great challenge. Due to the complexity of understanding visual patterns, precise detection has yet to be made. This lack of precise detection has led to the increasing demand for image processing, pattern recognition, machine learning, and machine vision to achieve sophisticated detection and classification of plant diseases. During studies conducted for plant disease classification, images were generated that could be employed for the development of the algorithm. This process involves excessive process time, development time, and execution time. Thus, for this purpose, image processing techniques are utilzied for the development of such a system which could classify and rule out plant disease

\section{LITERATURE REVIEW}

Due to the importance of plant health management universally, plant disease detection has attracted many researchers in this field for finding different methods to address this concern. As shown in Table 1. there could be many similarities between fungal and bacterial diseases.

An interesting method is discussed by Tamilselvi and Kumar [5] which makes the utlization of the artificial neural network (ANN). The Gabor filter was employed for feature extraction in the study, ANN which then classifies different plant diseases based on texture, colour and feature. However, the recognition rate could be increased.

In a study, Prabira [6] developed a technique employ histogram matching. This method was based on edge detection and colour feature. The training was performed by utilizing the layer separation technique. This technique classifies the image into RGB (Red, Blue, and Green) images. The colour cooccurrence was achieved by utilizing a Spatial Gray-level Dependence Matrices. There exist many techniques for classification of data, among these classification techniques, two were chosen for inspection in terms of accuracy which are (i) the decision tree and (ii) regression tree while studying on immature peach detection [7]. The random forest resembles that of a summing different decision trees which were obtained via an identical dataset having identical training points. The decision tree aims to reduce variation which occurs in the decision tree. The utlization of this classifier and others have been studied and analyze in detail [8]. Working on spot segmentation in plant disease was also performed for the identification of plant disease [9]. This study was conducted by comparing different colour spaces, namely as CIELAB, $\mathrm{HSI}$, and $\mathrm{YCbCr}$. A median filter was applied to smoothen the image, followed by the Otsu method on colour components and the calculation of the threshold lead to the detection of the diseased spot. Although some noise/unwanted signals were generated but were removed by employ CIELAB colour model. Most of the research conducted digital cameras with an optical axis which is perpendicular to the plane of the leaf. A study has also employed android mobile to capture images, the images were taken at a fix distance [10]. The images generated in a controlled environment are comparatively more suitable to work with. The result of the system greatly depends on the background and other distortion [11]. However, distortion removal could improve image quality making it easy for further processing. While analyzing the colour space conversion (CSC) techniques like hue, saturation, and value $\mathrm{HSV}$, it was noted that the CSC has a resembles that of a human sensing property [12]. A similar technique to HSV is HIS, where I stand for intensity [13]. Other CSC includes YCbCr, Hue-Max-Min-Diff, CIE 1976 L*a*b*, RGB, CIE 1976. Subsequent to CSC, desired enhancement is performed to achieve filtering for sharpening. In litrature, some commonly employed filters are neighbourhood means [14], spatial low pass filter, and frequency low pass filter. Complex computing methods such as fuzzy logic, genetic programming, and artificial neural networks [15] are also in practice. These complex computational methods require an input training data set for problem solving. Image acquisition is one of the most curial part for the system accuracy, as it depends on image samples, which are required for classifier training. Researchers have utlized known databases such an IPM Images, PlantVillage Images, and APS Image database [16]. The thermal imaging approach has also been implemented for finding plant diseases, the variation of tissue temperature has been studied and accurately detected by thermal images. The results generated were under a controlled environment [17].

Table 1. Fungal and bacterial disease signs and symptoms

\begin{tabular}{cccc}
\hline Fungal Disease (signs) & Fungal Disease (symptoms) & Bacterial Disease (signs) & Bacterial Disease Symptoms \\
\hline Leaf Rust & Leaf Spot & Bacterial Ooze & Mosaic Leaf Pattern \\
Stem Rust & Birds Eye Spot on Berries & Water Soaked Lesion & Crinkled Leaves \\
White & Chlorosis & Bacterial Streaming in Water from a Cut Stem & Yellowed Leaves \\
\hline
\end{tabular}


Interesting research was performed utilizing a fuzzy classifier, principal of grouping both negative and positive data set points into fuzzy groups [18]. These groups are then given a membership function defined by the truth value. A novel approach is presented by Kusumo et al. [19] this approach had aimed to identify the infection in wheat crop digital images. The data set is provided with both positive and negative images. The classifier gave an accuracy rate of $88 \%$ for positive (healthy) and $56 \%$ for a negative (unhealthy). KNN Classifier the K Nearest Neighbors is another simple classifier that employed a simple technique of storing all available cases and classifies new cases based on a similarity measure. The K-Nearest Neighbors is applied due to its pattern recognition, statistical estimation, and classification for machine learning. An approach was made for identifying diseases in sugarcane. Image processing was performed for feature extraction and then KNN classifier for classification [20]. Support Vector Machine (SVM) a technique that makes classes of the data point in such a way that each class as a similarity with the other data points of the same class, thus classifying or grouping similar data in a signal class. This study showed that image processing techniques could be employed to determine the level of nitrogen in the plant through leaf image. The study worked with pixels and SVM. Features were extracted and compared to determine if the leaf image was a high or low level of nitrogen. This research has improved plant health monitoring [21]. The novel study manipulated techniques of artificial intelligence algorithms and combined machine and deep learning to determine the evolution of COVID-19 keeping in view the of protective tools and confinement. The AI system developed showed that the chances of exposure to the virus could be reduced by $80 \%$ in the future [22]. Image processing techniques have also been applied in the field of Ophthalmology. A study suggested detecting glaucoma (a leading eye disease that results in loss of vision) by incorporating deep learning techniques and applying the Convolution Neural Network (CNN). The study was able to get encouraging results [23].

It is important to note, the database needs to be extended to maximize accuracy. Few diseases have been discussed or are limited to only a special kind of plant this could be countered if the dataset and data points are extended. Methods developed and discussed are novel and interesting; however, the implementation falls short in accuracy for some cases; hence, more feature optimization is needed and training samples for predicting the disease and improving the accuracy rate.

\section{PROPOSED METHOD}

A method is presented for automatic classification and localization utilizing the k-means cluster and a multiclass SVM. In general, most classifiers such as Neural Networks tend to minimize reducing the empirical risk or training error. The SVM [24] Is built on a different technique; working on the principle of reducing the empirical risk, the SVM operates on structural risk minimization. This process is executed by minimizes the upper boundary on the generalized error. Training an SVM is considered as solving a linearly constrained Quadratic Program (QP).

The proposed method is fully automatic, the accuracy is enhanced and time efficient. The developed method makes incoporates different techniques to achieve the objective of this research. The flow graph is shown in Figure 3. The method performs image acquisition, the input is then pre-processed. This step involves image smoothing, image enhancement, and contrast stretching. The result of the pre-processed image defines the image properly and makes it suitable for making clusters. The clusters would define three ROI (region of interest) by examining the colour and pattern of the given image. This would result in the grouping of three clusters. These three clusters would be of different colour and texture represented such that each cluster would have similarities with its group.

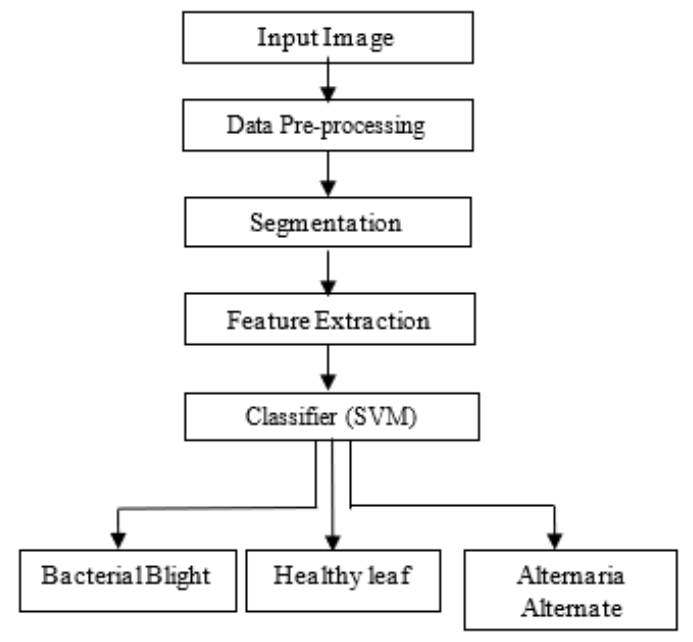

Figure 3. Proposed methodology

The three-cluster defined: One cluster was selected which was utlized for localization and determining the spread percentage. The classifier is developed incorporating a multiclass support vector machine (SVM) which consist of more than 3500 data points for each of the image i.e. healthy leaf, alternate and bacterial blight, resulting a total of more than 10,000 data points to train. The database stores new images and learn with every experiment that is performed. The images which are analyzed by the algorithm are stores in the database for the classier to train on new data points for better classification. The steps are discussed in detail to achieve the objective.

The first step (as shown in Figure 3), was to acquire proper images for establishing the dataset. An image capturing device was utlized to capture the images and store it in a data storing device for developing a proper data set to identify plant diseases. The images were first taken in a controlled environment. This process was done to eliminate any unwanted signals that may disturb the process of building up an accurate dataset. A total of 1000 images were found sufficient to classify the plant diseases respectively, although increasing it would increase the accuracy. The dataset was thus augmented, enhancing the data points from 1000 to 10,000. It was designed for the following, sets rotation, rescales value, shear range, zoom range, horizontal flip measure, width shift range, and height shift range. The second step involves pre-processing, which performs contrast stretching, image smoothing, and enhancement techniques. The pre-processed image is arranged in such a manner that the image would provide more data and information that could be utlized to further extract information (features). It is to note that this step has an important role as it enhances the diseased region of the plant when compared to the original image. While performing this step, it is important to (a) reduce noisy background, (b) applying the best contrast between leaf and background and (c) variation of light intensity 
must be kept ideal. These aspects affect the outcome of a vision-based system. The pre-processed image of a leaf is segmented employing the K-means clusters which create three different clusters of the input image. The clusters represent the images with three different clusters. The k-means clusters generate groups of pixels in which each pixel has some similarity with one another, three different clusters based on colour and texture. Figure 4 represents three clusters formed which have different segmented region but share similar texture or colour.

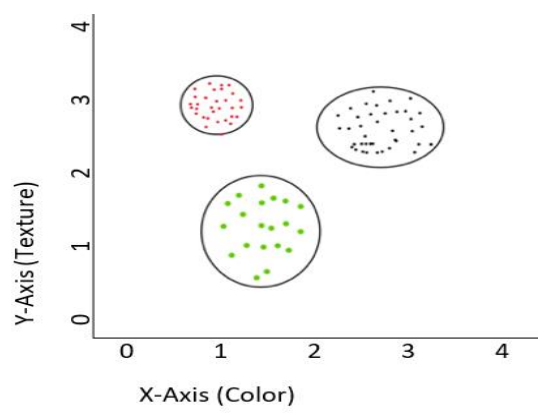

Figure 4. Cluster grouping

The cluster is an important aspect of the study as it evaluates the features, groups similar features into groups, also utilized for feature extraction and further processing. The clusters depend greatly on the quality of the image provided along with Hight, width, colour, texture, angle of the image capture. These clusters are given to the classifier for localization employing the k-means cluster, the image is then processed for feature extraction. However, the splitting effect depends on feature extraction. If the extracted features provide enough characteristics then the splitting effect would have a clear division. While extracting features the following are considered, as (i) leaf size (ii) leaf colour (iii) leaf shape, and (iv) Pattern recognition/Irregular surface. These four features are extracted from the cluster image defining ROI. These four features are very important for determining the state of any leaf. In this paper, the SVM has been employed to train and determine if the leaf is healthy or unhealthy. This classifier requires training data. The training data is provided by feature vectors and data output instances termed as labels, these features and labels train the SVM known as the prediction model. The SVM received the pre-processed image as input. It works to minimize the error function. The mathematical representation of the minimization of the error function is shown in Eq. (1).

$$
\frac{1}{2} w^{T} w+c \sum_{i=I}^{N} \delta_{i}
$$

Machine learning classifier has a limitation, it depends on other external factors. A few of these factors are dataset, feature extraction, feature storing, data analysis and image processing techniques. Due to the complexity of machine learning, there are many challenges when studying and working with it. the major problems faced are decision making interpretability, quality of data issue, lack of transparency, security and safety issue.

\section{EXPERIMENTATION}

The experimentation phase for detection of plant disease through digital imaging was performed on MATLAB R2018a and operated on the core i5 processor. The Camera resolution was set at $640 \times 480$. The input image was captured using an android or apple device, although for creating the dataset, a controlled environment was established which kept the light intensity and external factor under control. These captured images were incoporated for proving data points and dataset for the classifier. The develpved algorithm is a combination of image enhancement, clustering, and image classification employing a multi-class SVM. The plant disease detection after the selection of clusters (ROI) was at a estimate time of 0.1-0.4 seconds.

The condition that was implemented during the experimentation was as follows: The first step when starting the experimentation was to adjust the image according to the requirement of the algorithm. This means removing any unnecessary data of the image that would delay the operation and also crate delay in the computation processing time. The pixels were reduced or increase depending on the image resolution, for this the dots per inch (DPI) of the images were set to 240 per image, this implies that the images had a height of 240 pixels and weight 240 pixels respectively. This provided the algorithm with uniform datapoints regarding pixels, for performing the training. Any image lower to above 240 was set to 240 first and then added to the data set for performing training.

The lighting condition was also set to a uniform. Three sets of lighting were provided, bright sunlight, torchlight, and room light. These lightings were to be set so that they may not create any shadows on the leaf. This was very important as these shadows results in error in detection. We have to provide the algorithm with ideal samples to train on. While working with the torch and flashlight, an important parameter of angle is also involved. These angles had two axes termed as pan and tilt. Although, for each image, the pan and tilt angles were found to be different, however, ideally the pan and tilt angles should be $180^{\circ}$ for pan and tilt should be $90^{\circ}$.

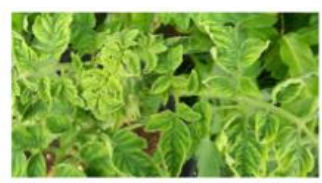

(a)

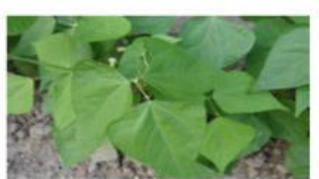

(b)

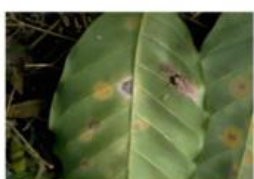

(c)
Figure 5. (a) Tomato Leaves, (b) Guava Leaves, (c) Coffee Leaves

There were two stages of experiments, portioned into two subsections: the first subsection was in a laboratory experiment and the second subsection on the ground experiment. The laboratory experiment was executed in a controlled environment. This provided an ideal situation for the image processing techniques to perform adequately. The major parameter to consider is for the illumination to be kept properly. The in-laboratory experiment was performed for six months on different plant leaves. The algorithm exhibited imitation which was improved employing advanced image processing 
techniques. These experiments were performed on different leaves. Due to the controlled environment, there was no distortion in the image which needed to be eliminated and thus did not provide means for false detection. However, during the second subsection, the experiment was performed on the ground, in the field. These experiments did not have a controlled experiment and were performed under different light and weather conditions. The on-ground experiments were performed for six months and were tested on different plants and different fields. The name of the plant on which these experiments were conducted is displayed in Figure 5.

During experiments, one of the challenges was environmental conditions and removal of distortion. During very low illumination, the leaf could not be detected by the algorithm; thus, a flashlight was installed for capturing images. This provided clear and accurate images for the algorithm. The experimentation had an accuracy ratio of $96.03 \%$, this was envaulted by the formula in Eq. (2).

$$
\text { Accuracy }=\frac{T P+T N}{P+N}
$$

The experimentation was conducted over one year. These experiments were divided into two subsections in-laboratory and on ground experimentation. During the two subsections of the experimentation, it was found that the classifier depends greatly on the input images and dataset. Henceforth, to improve the classifier results, the input images and results were stored, this improved the result of the classifier with every experiment performed due to data being updated with every experiment. The images given to the algorithm are stored in the dataset, the farmer/operator provides algorithm with raw data, images, and sample points which are classified by the algorithm.

It is to be noted that the SVM requires lesser samples for training and evaluation and due to it working on structural risk, the algorithm has been more time effect and required less computational processing delay time.

\section{RESULTS AND ANALYSIS}

The proposed method has been developed and experimented on a different plant leaf (three class). The experiments were repeated with a different condition and repeated over a time interval of one year on different plants. The database had data points exceeding ten thousand results are shown in Table 2 for presentation.

Furthermore, a second accuracy test was also applied to the classifier, precision, and recall. The precision, and recall method is employed for testing the accuracy in pattern recognition and machine learning classifier. The values which are positive predicted values, terms as precision, is a fraction between relevant instances and retrieve instances and the sensitivity of this is called recalled, is the fraction of the amount of total of relevant instances that were retrieved. Thus, they are based on the measure of relevance. The formula of precision and recall are as followings:

$$
\begin{gathered}
\text { Precision }=\frac{\{\text { Relevant }\} \cap\{\text { Retrieve }\}}{\{\text { Retrieve }\}} \\
\text { Recall }=\frac{\{\text { Relevant }\} \cap\{\text { Retrieve }\}}{\{\text { Relevent }\}}
\end{gathered}
$$

The precision and recall recorded are $94.957 \%$ and $94.843 \%$. $\mathrm{F} 1$ is Here F1 represents the overall performance which is
94.9\%. In summary, it is the harmonic mean of the P and R. The execution time of the algorithm was within four seconds. An image has been taken and provided to the algorithm to apply image pre-processing techniques and image enhancement to upgrade the image representation. After the pre-processing and image enhancement were conducted the image was then segmented into three clusters. The operator must select the cluster of the pattern that is required to be evaluated. After the selection, the segmented region is passed to SVM to classify the condition of the leaf. The cluster for the figures shown in Figure 6 for healthy leaf, Alternaria Alternate Alternaria and Bacterial Blight respectably. While these results were encouraging enough, the accuracy rate of the classifier needed to be established, thus; a confusion matrix was employed to determine the accuracy rate of the classifier.

Table 2. Results of experimentation

\begin{tabular}{cccc}
\hline $\begin{array}{c}\text { S. } \\
\text { No }\end{array}$ & $\begin{array}{c}\text { Image } \\
\text { Acquired }\end{array}$ & $\begin{array}{c}\text { Pre-Processed } \\
\text { Image }\end{array}$ & Result \\
\hline & & & \\
& & &
\end{tabular}

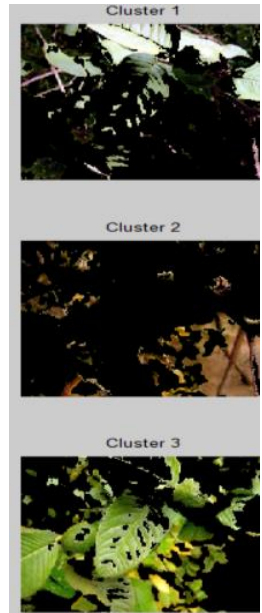

(a)

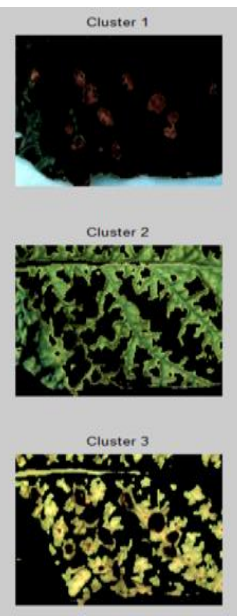

(b)

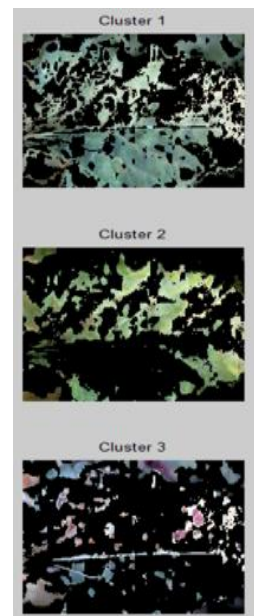

(c)
Figure 6. (a) Healthy leaf, (b) Alternaria Alternate, (c) Bacterial Blight

These confusion matrices are typically utlized to check the accuracy of the performance of any classifier. The confusion matrix has only two possible cases for prediction, ' 1 ' and ' 0 ', where ' 1 ' indicates as the disease exists and ' 0 ' indicates the opposite, disease does not exist. The classifier was employed to determine the state of plant leaves. Each class having at least 2500 approximate samples for testing and evaluating. According to the results generated by the confusion matrix, an accuracy rate of $95 \%$. A better understanding of the confusion matrix is explained in Table 3. 
Table 3. Confusion matrix

\begin{tabular}{c|c|c|c}
\hline Predicted & True Positive & True Negative & \multirow{2}{*}{5,003} \\
\cline { 2 - 3 } Positive & 4,789 & 214 & \\
\hline Predicted & 211 & 4,786 & 4,997 \\
\cline { 2 - 3 } Negative & 5,000 & 5,000 & 10,000 \\
\hline
\end{tabular}

Table 4. Affected area in $\%$ age

\begin{tabular}{ccc}
\hline S No. & Name & Spread (in \%age) \\
\hline $\mathbf{1}$ & Alternaria Alternate & $15.0113 \%$ \\
$\mathbf{2}$ & Bacterial Blight & $15.0077 \%$ \\
\hline
\end{tabular}

The detection of the disease is an important aspect of this study; however, localization was also required for understanding the state of the plant; thus, a procedure was performed to analyses the surface of the leaf that has been affected by the disease. For this, it is important to examine the segmented region. The k-means segmentation provides three segments, the segmented region having the ROI minus the remaining region could be utlized to evaluate the diseased spread percentage. The results generated by the utlization of these techniques for disease spread are represented in a tabular form as shown in Table 4.

The table represents experiments performed on two plant leaves. The leaf could be classified by the algorithm as Alternaria alternate and bacterial blight. The algorithm was then employed to evaluate the percentage spread. The results were shown as $15.0113 \%$ for Alternaria alternate and $15.0077 \%$ for bacterial blight.

\section{A COMPARISION FOR EVALUATION}

The method discussed by Reza et al. [25] and Narmadha and Arulvadivu [26] are limited to stem or paddy plants while the developed method (DM) is could oversee type on the plant as it analyses the texture of the disease. The established method was compared to some of the developed methods for better understanding as shown in Table 5.

Table 5. A comparison for evaluation

\begin{tabular}{ccccc}
\hline S. No & Method & Compatible & Techniques & Accuracy \\
\hline 1 & {$[25]$} & $\begin{array}{c}\text { Bacterial and Fungal } \\
\text { disease (for stem only) }\end{array}$ & $\begin{array}{c}\text { Colour Co-occurrence Method and } \\
\text { multi-class SVM }\end{array}$ & $86 \%$ \\
\hline 2 & {$[26]$} & Bacterial disease only & Fuzzy classification, SVM & $94.70 \%$ \\
\hline 3 & $\begin{array}{c}\text { Developed } \\
\text { Method }\end{array}$ & $\begin{array}{c}\text { Bacterial and Fungal } \\
\text { Disease (entire plant) }\end{array}$ & $\begin{array}{c}\text { K-means segmentation, Multi-class } \\
\text { SVM }\end{array}$ & $95 \%$ \\
\hline
\end{tabular}

The method employs eight different plants and has produced encouraging results. The method discussed by Reza et al. [25] employed the SVM classifier and utlized of the colour cooccurrence. The classifier employed was a support vector machine which gave an accuracy of $86 \%$. Similarly, Narmadha and Arulvadivu [26] made utilization of fuzzy classification and SVM for detecting paddy plant-oriented diseases, the method did generate high accuracy up to $94.70 \%$ working only on paddy plant. The developed method worked on bacterial and fungal oriented diseases utilizing $k$-means segmentation for segmenting similarities into one cluster and SVM for classifying the data accordingly. The accuracy for DM is $95 \%$, a graphical representation is shown in the Figure 7.

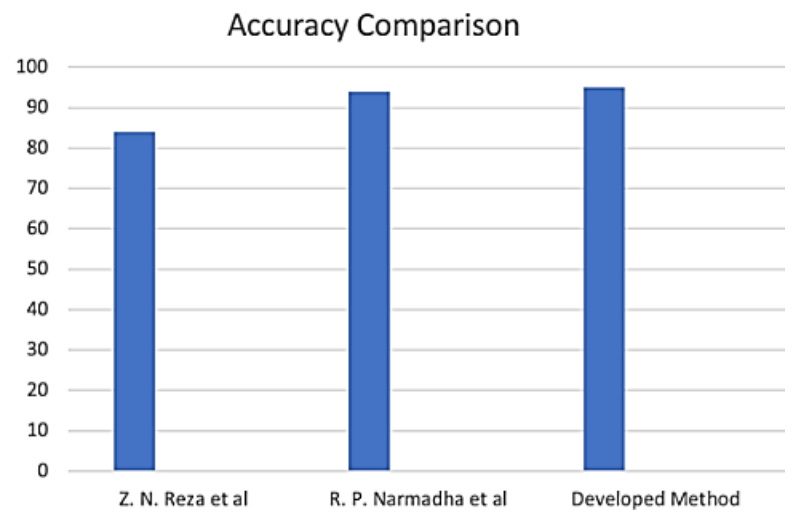

Figure 7. Accuracy comparison

Machine vision-based technologies and detection has proven to be faster, accurate, and cheap rather than manual detection. It is, therefore, the reason this approach was employed to distinguish between healthy and diseased plants. The system could be utlized in real-time to help assist the farmers. The DM has a potential of image classification into three classes, (i) healthy (ii) Alternaria alternate, and (iii) bacterial blight. This research has made possible utilizing a dataset consisting of data points and images that were more than 10,000 along with other advanced image processing techniques. This approach would help and assist the existing methods for yield loss prevention and plant health monitoring. With the advancement in computational power and technologies, plant pathology would be approached by employing computer-aided diagnoses in the future.

\section{CONCLUSION AND FUTURE WORK}

In this study, experiments were performed over 10,000 images, varying with light intensity and location. This approach was successful in determining three classes, i.e. healthy leaf, Alternaria Alternate, and Bacterial Blight. The developed algorithm is a combination of image enhancement, clustering, and image classification employing a multi-class SVM. The method was compared with already established work and an accuracy rate of $95 \%$ was recorded. The developed method has the capability in the identification of three types of disease in a plant.

It would be interesting to incorporate another open-source software tool such as Python, for a comparison between multiple software for efficient computation.

\section{REFERENCES}

[1] Sahay, A., Chen, M. (2016). Leaf analysis for plant recognition. In 2016 7th IEEE International Conference on Software Engineering and Service Science (ICSESS), Beijing,

China

pp.

914-917. 
https://doi.org/10.1109/ICSESS.2016.7883214

[2] Anthonys, G., Wickramarachchi, N. (2009). An image recognition system for crop disease identification of paddy fields in Sri Lanka. In IEEE International Conference on Industrial and Information Systems, Peradeniya, Sri Lanka, pp. 403-407. http://dx.doi.org/10.1109/ICIINFS.2009.5429828

[3] Asfarian, A., Herdiyeni, Y., Rauf, A., Mutaqin, K.M. (2009). Paddy diseases identification with texture analysis using fractal descriptors based on Fourier spectrum. In IEEE International Conference on Computer, Control, Informatics and Its Applications IC3INA, Jakarta, Indonesia, pp. 77-81. http://dx.doi.org/10.1109/IC3INA.2013.6819152

[4] Bagde, S., Patil, S., Patil, P. (2015). Artificial neural network based plant leaf disease detection. International Journal of Computer Science and Mobile Computing, 4(4): 900-905. https://doi.org/10.15680/IJIRCCE.2019. 0705005

[5] Tamilselvi, P., Kumar, K.A. (2017). Unsupervised machine learning for clustering the infected leaves based on the leaf-colours. In Third International Conference on Science Technology Engineering \& Management (ICONSTEM), Chennai, India, pp. 106-110. http://dx.doi.org/10.1109/ICONSTEM.2017.8261265

[6] Ayaz, M., Ammad-Uddin, M., Sharif, Z., Mansour, A., Aggoune, E.M. (2019). Internet-of-things (IoT)-based smart agriculture: Toward making the fields talk. IEEE Access, 7: 129551-129583. http://doi.org/10.1109/ACCESS.2019.2932609

[7] Shruthi, U., Nagaveni, V., Raghavendra, B.K. (2019). A review on machine learning classification techniques for plant disease detection. In 5th International Conference on Advanced Computing \& Communication Systems (ICACCS), Coimbatore, India, pp. 281-284. http://dx.doi.org/10.1109/ICACCS.2019.8728415

[8] Guo. W., Zeng, T., Huang, T., Cai, Y. (2020) Disease cluster detection and functional characterization. IEEE Access, 8: 141958-141966. http://doi.org/10.1109/ACCESS.2020.3013666

[9] Ashourloo, D., Matkan, A.A., Huete, A., Aghighi, H., Mobasheri, M.R. (2016). Developing an index for detection and identification of disease stages. IEEE Geoscience and Remote Sensing Letters, 13(6): 851-855. http://doi.org/10.1109/LGRS.2016.2550529

[10] Pardede, H.F., Suryawati, E., Sustika, R., Zilvan, V. (2018). Unsupervised convolutional autoencoder-based feature learning for automatic detection of plant diseases. In International Conference on Computer, Control, Informatics and its Applications (IC3INA), Tangerang, Indonesia, $\quad$ pp. 158-161 http://dx.doi.org/10.1109/IC3INA.2018.8629518

[11] Valdoria, J.C., Caballeo, A.R., Fernandez, B.I.D. (2019). iDahon: An android based terrestrial plant disease detection mobile application through digital image processing using deep learning neural network algorithm. In 4th International Conference on Information Technology (InCIT), ChonBuri, Thailand, pp. 94-98. http://dx.doi.org/10.1109/INCIT.2019.8912053

[12] Park, H., JeeSook, E., Kim, S. (2018). Crops disease diagnosing using image-based deep learning mechanism. In International Conference on Computing and Network Communications (CoCoNet), Astana, Kazakhstan, pp. 23-26.
http://dx.doi.org/10.1109/CoCoNet.2018.8476914

[13] Oerke, Steiner, U., Dehne, H.W., Lindenthal, M. (2006). Thermal imaging of cucumber leaves affected by downy mildew and environmental conditions. Journal of Experimental Botany, 57(9): 2121-2132. https://doi.org/10.1093/jxb/erj170

[14] Janarthan, S., Thuseethan, S., Rajasegarar, S., Lyu, Q., Zheng, Y., Yearwood, J. (2020). Deep metric learning based citrus disease classification with sparse data. IEEE Access, $\quad 8$ : 162588-162600. https://doi.org/10.1109/ACCESS.2020.3021487

[15] Prakash, R.M., Saraswathy, G.P. (2017). Detection of leaf diseases and classification using digital image processing. In International Conference on Innovations in Information, Embedded and Communication Systems (ICIIECS), Coimbatore, India, pp. 1-4. http://dx.doi.org/10.1109/ICIIECS.2017.8275915

[16] Madallah, A., Saad, A., Ghany, E., Shehab, S., Abdulaziz. (2019). An efficient deep learning model for olive diseases detection. International Journal of Advanced Computer Science and Applications, 10(8): 486-492. http://dx.doi.org/10.14569/IJACSA.2019.0100863

[17] Ghiasi, M., Amirfattahi, R. (2013). Fast semantic segmentation of aerial images based on colour and texture. In 8th Iranian Conference on Machine Vision and Image Processing (MVIP), Zanjan, Iran, pp. 324-327. http://dx.doi.org/10.1109/IranianMVIP.2013.6780004

[18] Kobayashi, K., Tsuji, J., Noto, M. (2018). Evaluation of data augmentation for image-based plant-disease detection. In IEEE International Conference on Systems, Man, and Cybernetics (SMC), Miyazaki, Japan, pp. 2206-2211. http://dx.doi.org/10.1109/SMC.2018.00379

[19] Kusumo, B.S., Heryana, A., Mahendra, O., Pardede, H.F. (2018). Machine learning-based for automatic detection of corn-plant diseases using image processing. In 2018 International Conference on Computer, Control, Informatics and its Applications, Bandung, pp. 93-97. http://dx.doi.org/10.1109/IC3INA.2018.8629507

[20] Weizheng, S., Yachun, W., Zhanliang. C., Hongda, W. (2008). Grading method of leaf spot disease based on image processing. In IEEE International Conference on Computer Science and Software Engineering, Wuhan, China, pp 491-494. http://dx.doi.org/10.1109/CSSE.2008.1649

[21] Djawad, Y.A., Rehman, H., Jumadi, O., Tufail, M., Anwar, S. Bourgougnon, N. (2020). Discrimination of nitrogen concentration of fertilized corn with extracted algae and polymer based on its leaf colour images. Ingénierie des Systèmes d'Information. 25(3): 303-309. https://doi.org/10.18280/isi.250303

[22] Ksantini, M., Kadri, N., Ellouze, A., Turki, S.H. (2020). Artificial intelligence prediction algorithms for future evolution of COVID-19 cases. Ingénierie des Systèmes d'Information. 25(3): 319-325. https://doi.org/10.18280/isi.250305

[23] Kanagala, H.K., Krishnaiah, V.V.J. (2020). Detection of glaucoma using optic disk segmentation based on CNN and VAE models. Ingénierie des Systèmes d'Information. 25(3): 371-376. https://doi.org/10.18280/isi.250312

[24] Garcia, C., Tziritas, G. (1999). Face detection using quantized skin colour face detection using quantized skin colour regions merging and wavelet packet analysis. IEEE Transactions on Multimedia, 1(3): 264-277. https://doi.org/10.1109/6046.784465 
[25] Reza, Z.N., Nuzhat, F., Mahsa, N.A., Ali, M.H. (2016). Detecting jute plant disease using image processing and machine learning. In 3rd International Conference on Electrical Engineering and Information Communication Technology (ICEEICT), Dhaka, Bangladesh, pp. 1-6. http://dx.doi.org/10.1109/CEEICT.2016.7873147

[26] Narmadha R.P., Arulvadivu, G. (2017). Detection and measurement of paddy leaf disease symptoms using image processing. In International Conference on Computer Communication and Informatics (ICCCI), Coimbatore, pp.

http://dx.doi.org/10.1109/ICCCI.2017.8117730

\section{NOMENCLATURE}

c w T $\delta$ i $\mathrm{N}$

C

Capacity constant Vector of coefficients Parameters to manage non-separable data (inputs) Index

Test images

Factor which penalize the error 